\title{
All-Optical RZ Data Conversion With Temporally and Spectrally Gain-Sliced Semiconductor Optical Amplifier Via Broadband Optical Injection
}

\author{
Gong-Ru Lin, Senior Member, IEEE, Kun-Chieh Yu, and Mu-Chieh Lo
}

\begin{abstract}
We investigate the effect of the backward injecting linewidth on the rise/fall time, duty cycle, and frequency chirp of the $10 \mathrm{~Gb} / \mathrm{s}$ format-converted pulsed return-to-zero $(\mathrm{RZ})$ data stream from the traveling-wave semiconductor optical amplifier (TWSOA) with its gain profile temporally and spectrally reshaping by a backward injected single- or multimode inverse-opticalcomb. The rising time remains almost unchanged; however, the falling time was monotonically reduced by increasing injection power. The evolution of full-width at half-maximum with injection power exhibits similar trend with falling time. In comparison with single-mode inverse-optical-comb injection, the multimode injection exhibits frequency-chirp reduction and better ON/OFF extinction performances. Under the injection of multi- and single-mode inverse-optical-combs into the TWSOA at extremely high gain condition, the multi- and single-mode injection converted pulsed $\mathrm{RZ}$ data pulsewidths are 32 and 31 ps with corresponding peak-topeak chirps of 10 and $12 \mathrm{GHz}$, respectively. The chirp can abruptly be reduced to $1.2 \mathrm{GHz}$ by reducing the TWSOA biased current to $100 \mathrm{~mA}$.
\end{abstract}

Index Terms-All optical, chirp, data conversion, NRZ to RZ, optical injection, semiconductor optical amplifier, $10 \mathrm{Gbit} / \mathrm{s}$.

\section{INTRODUCTION}

A LL-OPTICAL modulation format conversion between data streams with different formats in traveling-wave semiconductor optical amplifier (TWSOA) based subsystems has emerged as an important topic of research recently [1][30]. By using special techniques such as gain clamping, optical injection, cross-phase modulation, nonlinear polarization rotation [1]-[3], [5]-[7], [13], four-wave mixing [23], crossgain modulation (XGM) [20], bandpass filtering [30], loopmirror [15], [17], [19], and interferometer [8], [21], [29], the transformation between non-return-to-zero (NRZ) and returnto-zero (RZ) formats or between NRZ ON-OFF keying (NRZOOK) and RZ quadrature phase shift keying (RZ-QPSK) formats can be implemented. Other speculated functions including all-optical clock-recovery [9], [10], [12], [16], [17], [22], 3R regeneration [11], and wavelength-division multiplexing (WDM) multicasting [31] have also been involved together with the

Manuscript received November 14, 2007; revised January 19, 2008. This work was supported by the National Science Council of Republic of China under Grants NSC96-2221-E-002-099 and NSC96-2752-E-009-008-PAE.

The authors are with the Institute of Photonics and Optoelectronics, National Taiwan University, Taipei 106, Taiwan, R.O.C., and also the Department of Electrical Engineering, National Taiwan University, Taipei 106, Taiwan, R.O.C. (e-mail: grlin@ntu.edu.tw; hevin1031@gmail.com; b92901081@ntu.edu.tw).

Color versions of one or more of the figures in this paper are available online at http://ieeexplore.ieee.org.

Digital Object Identifier 10.1109/JSTQE.2008.918245 data format converter to extend the feasibility of such device for versatile applications. To date, the operational bit rates extending from 10 to $40 \mathrm{~Gb} / \mathrm{s}$ have previously been demonstrated [2], [3], [6], [13]-[17]. Recently, the backward inverseoptical-comb injection was proposed as a novel XGM technique for mode-locking TWSOA-based fiber ring lasers, which benefits from the precise gain control in time domain and the high optical modulation bandwidth of the TWSOA [31]. Later on, similar technology was employed for all-optical data transformation between NRZ and RZ to integrate wavelength division multiplexing and optical time division multiplexing architectures [32]-[37]. In particular, a noninverting wavelength shifting scheme was successfully demonstrated [28], [36]. However, the efforts on some featured parametric analyses such as extinction ratio enhancement and dynamic chirp control were less paid for such system [24], [25], [31]-[36]. In this paper, we theoretically and experimentally compare the effect of the backward injecting linewidth on the rise/fall time, duty cycle, and frequency chirp of the wavelength-preserved and format-converted pulsed $\mathrm{RZ}$ data stream at $10 \mathrm{~Gb} / \mathrm{s}$ from the TWSOA with its gain profile temporally and spectrally slicing by the backward injected single- or multimode inverse-optical-comb. We discuss the spectral and temporal domain response of the TWSOA-based NRZto-RZ data converter with use of single- or multimode inverseoptical-comb injection techniques. In the first part, we discuss the effect of single- or multimode on the converted RZ pulse shape, extinction ratio and bit-error-rate (BER) performances. In the second part, we discuss the converted RZ pulsewidth and chirp characteristics under the backward single- or multimode inverse-optical-comb injections. Theoretical simulations on the converted pulseshape and corresponding chirp are also provided to elucidate the influence of the inverse-optical-comb pulsewidth.

\section{EXPERIMENTAL SETUP}

The TWSOA based NRZ-to-RZ format converter is illustrated in Fig. 1. The TWSOA with maximum gain at $1530 \mathrm{~nm}$ and amplified spontaneous emission linewidth of $35 \mathrm{~nm}$ is dcbiased (dc voltage biased) well beyond its transparent condition (typically, $\mathrm{I}_{\mathrm{dc}} \cong 350 \mathrm{~mA}$ ). To temporally reshape the TWSOA gain window, the single- or multimode Fabry-Perot laser diode (FPLD) inverse-optical-comb is generated by passing a continuous-wave $(\mathrm{CW})$ laser diode (LD) output through a Mach-Zehnder intensity modulator (MZM) driven by an amplified electrical comb signal and a dc offset voltage to make the MZM operating at nonlinear modulation regime. In our 


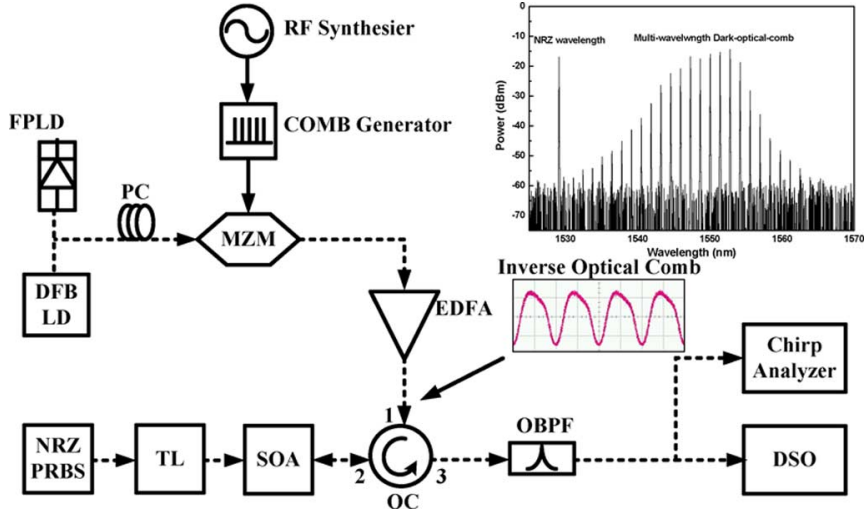

Fig. 1. Experimental setup. DSO: digital sampling oscilloscope; EDFA erbium-doped fibre amplifier; OBPF: optical bandpass filter; OC: optical circulator; PC: polarization controller; TL: tunable laser. Electrical path: solid line. Optical path: dash line.

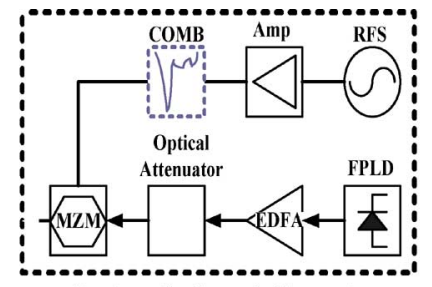

Dark-optical-comb Generator

(a)

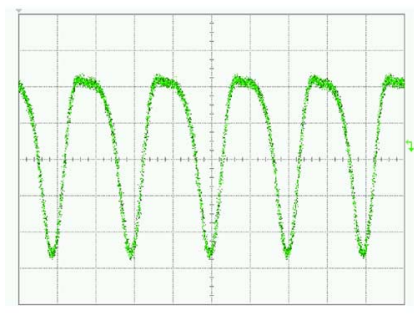

(c)

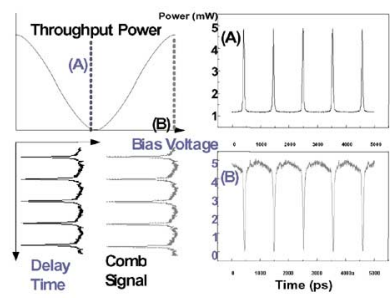

(b)

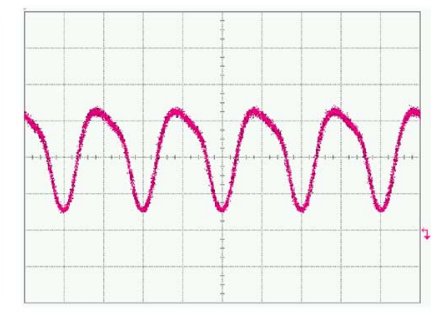

(d)
Fig. 2. (a) Illustration of inverse-optical-comb generator. (b) MZM-biased condition for normal-optical-comb and inverse-optical-comb generation. (c) $10 \mathrm{GHz}$ electrical comb. (d) $10 \mathrm{GHz}$ inverse-optical-comb traces.

approach, we need an inverse-optical-comb to inject the TWSOA for reshaping the incoming NRZ data stream into the pulsed RZ data. This requires an electrical pulse to drive the MZM for inverse-optical-comb regeneration. Currently, the commercial available electrical pulse comes from an electrical comb generator, which can only give a negative (or inverse) electrical pulse. A commercially available electrical comb generator activated by a $10-\mathrm{GHz}$ sinusoidal clock signal of $30 \mathrm{dBm}$ generates the inverse electrical pulse train of 30-ps pulsewidth, leading to the generation of an inverse-optical-comb (see inset of Fig. 1) from the nonlinearly dc-biased MZM. The typical output waveform of such a commercial electrical comb generator with $10-\mathrm{GHz}$ period is an inverse electrical pulse, as plotted in Fig. 2(c), which is activated by a $27-\mathrm{dBm}$ amplified $10-\mathrm{GHz}$ sinusoidal clock signal with a duty cycle of $70 \%$ output from the MZM. In this case, we have to detune the dc bias of the MZM in order to obtain inverse-optical-comb. Both the brightand inverse-optical-comb pulse trains can be generated from the MZM biased with the offset DV electrical comb at different dc-biased conditions [i.e., (A) or (B)], as shown in Fig. 2(b), respectively. The CW laser source with similar central wavelength at about $1550 \mathrm{~nm}$ is either a single-mode distributed feedback LD (DFBLD, Mitsubishi FU-68PDF) with a 3-dB linewidth of $1 \mathrm{MHz}$, or a multimode FPLD (NEC 5501EH) with 3-dB linewidth of $7.297 \mathrm{~nm}$. The corresponding spectra of the input optical NRZ data and backward injected inverse-optical-combs are shown in Fig. 3. In contrast to the normal-optical-comb with a small duty cycle, the inverse-optical-comb waveform exhibits an extremely large duty cycle, as shown in Fig. 2(d), which is like a quasi-CW optical signal, but transiently turned off within a extremely short duration of $<35 \mathrm{ps}$ at a repetition period of $100 \mathrm{ps}$. After propagating through an erbium-doped fiber amplifier (EDFA) with $20 \mathrm{~dB}$ gain and an optical circulator (OC), the inverse-optical-comb is used to backward inject, and then, periodically deplete the gain of TWSOA for implementing NRZ-toRZ data format conversion [38]. The incoming optical NRZ data stream is simulated by externally encoding a tunable laser with another MZM driven by a pseudorandom-bit-sequence (PRBS) data-stream generator. The average power, ON/OFF extinction ratio, and pattern length of the incoming optical NRZ PRBS at $1529.2 \mathrm{~nm}$ are $-15 \mathrm{dBm}, 12 \mathrm{~dB}$, and $2^{23}-1$, respectively. The input NRZ wavelength is setting at the vicinity of peak wavelength of the TWSOA around $1530 \mathrm{~nm}$. However, the inverse-optical-comb is injected at a wavelength of 1550$1555 \mathrm{~nm}$ longer than that of the input NRZ data stream, which facilitates to achieve a highest XGM for optimizing the ON/OFF extinction ratio of the converted RZ data stream [39]. Technically, the injecting inverse-optical-comb power is gradually increased to approximately $8-9 \mathrm{dBm}$ for saturating the TWSOA gain to achieve a narrow gain window with maximum ON/OFF extinction ratio. The dynamic frequency chirp at different inverseoptical-comb injection powers is real-time monitored with a chirp analyzer (Advantest Q7606B). In general, both the copropagating (forward) and counterpropagating (backward) injection schemes can also realize the RZ signal regeneration in our proposed scheme. In both the cases, the regenerated RZ signal would be mixed with the inverse-comb signal at the output port of the TWSOA based decision gate, which thus requires an additional bandpass filter to filter out the injected signal in order to obtain the regenerated RZ signal with a clean pedestal. We take counterpropagating (backward) injection scheme as an example in our experiment.

\section{RESUlTS AND DiscusSIONS}

\section{A. Theoretical Simulation}

In general case, the gain of TWSOA can be derived from the output electric field $E(z, t)$ of the input signal, which can be described using a partial differential equation proposed by Agrawal and Olsson [40] previously, that is, $\partial E / \partial z+$ $\left(1 / v_{g}\right)(\partial E / \partial t)=\left(1-j \alpha_{l}\right) g E / 2$ [41], [42], where the electrical field can be expressed as $E(z, t)=\sqrt{P_{\text {out }}(z, t)} e^{j \phi(z, t)}$ 


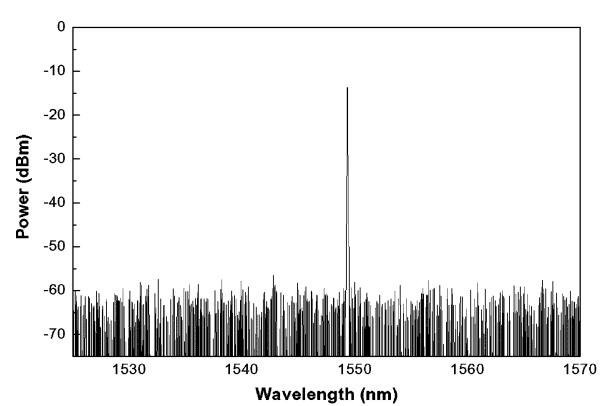

(a)

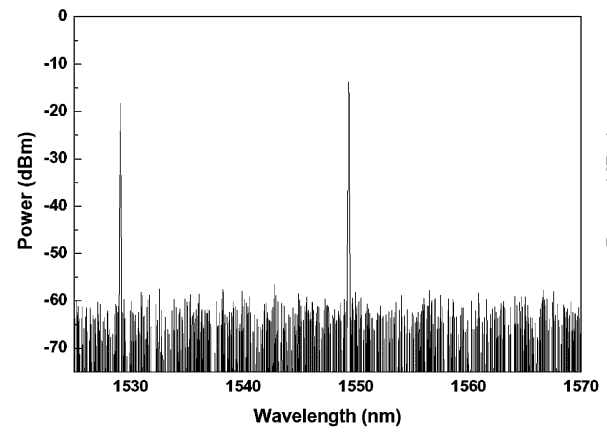

(c)

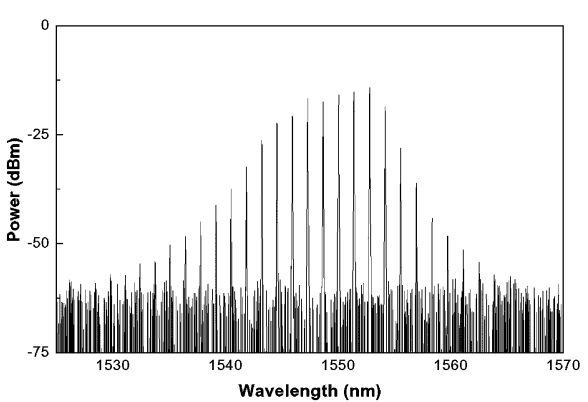

(b)

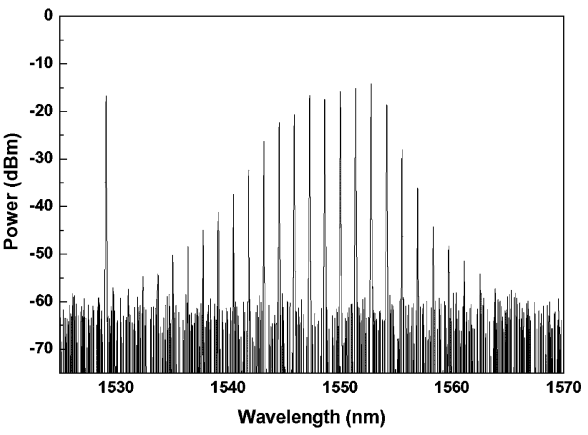

(d)

Fig. 3. (a) Lasing spectra of DFBLD. (b) Lasing spectra of FPLD. (c) Spectra of the input signal at $1530 \mathrm{~nm}$ and the single-mode inverse-optical-comb at $1550 \mathrm{~nm}$. (d) Spectra of the input signal at $1530 \mathrm{~nm}$ and the multimode inverse-optical-comb at $1550 \mathrm{~nm}$.

with $P_{\text {out }}(z, t)$ and $\phi$ denoting the signal power and phase, respectively, $v_{g}$ is the group velocity, and $\alpha_{l}$ is a constant usually referred to as the linewidth enhancement factor that couples the phase of the propagating signal to the gain coefficient of the semiconductor optical amplifier (SOA). Assuming that the gain of TWSOA exhibits a Lorentzian lineshape and is also a linear function of the spectral distribution of carrier density $(n)$ or carrier numbers $(N)$ [42], [43], [44], as described by

$$
g(\lambda, N)=\frac{a\left(n-n_{0}\right)}{1+\left(\lambda-\lambda_{N}\right)^{2} /\left(\Delta \lambda_{g}\right)^{2}}
$$

where $\Delta \lambda_{g}$ denotes as the 3-dB spectral linewidth of the TWSOA gain, $\lambda_{N}$ is the peak wavelength at carrier density $n, a$ is the differential gain coefficient, and $n_{0}$ is the carrier density required for transparency. To simplify the solution, the static time variable $t$ and spatial coordinate $z$ can be transformed into a time frame $\tau$ moving with the propagating signal, i.e., $\tau=t-z / \nu_{g}$. Such a transformation gives $\partial P_{\text {out }} / \partial z=g P_{\text {out }}$ and $\partial \phi / \partial z=-\alpha_{l g / 2}$, and the rate equation of carrier density can thus be expressed as

$$
\frac{\partial n}{\partial \tau}=\frac{I}{e d W L}-\frac{n}{\tau_{c}}-\frac{g P_{\mathrm{out}}}{d W h \nu}
$$

where $I$ is the biased current of TWSOA, $\tau_{c}$ is the spontaneous carrier lifetime, and $d W L$ is the volume $V$ of active region. With the gain coefficient $g$ approximating by $g=\Gamma a_{1}\left(n-n_{0}\right)$, and by substituting (1) into (2), the transient gain of TWSOA is described as

$$
\frac{d g}{d \tau}=\frac{g_{0}-g}{\tau_{c}}-\frac{g P_{\mathrm{out}}(\tau)}{E_{\mathrm{sat}} L}
$$

where $g_{0}$ is the small signal gain coefficient given by

$$
g_{0}=a_{1} n_{0}\left(\frac{I}{I_{0}}-1\right)
$$

the bias current required for transparency is

$$
I_{0}=\frac{e V n_{0}}{\tau_{c}}
$$

and the saturation energy is

$$
E_{\mathrm{sat}}=\frac{h \nu d W}{a_{1}} .
$$

If we assume $h(\tau)$ as the integrated gain at each point of the propagating pulse profile in which $h(\tau)$ is the integrated gain, $P_{\text {in }}(\tau)$ and $P_{\text {out }}(\tau)$ are the temporally varied input and output power functions of TWSOA, respectively, and the solution of $P_{\text {out }}(t)$ is assumed as

$$
P_{\text {out }}(\tau)=P_{\text {in }}(\tau) \exp [h(\tau)]
$$

with

$$
h(\tau)=\int_{0}^{\tau} g(z, \tau) d z .
$$

This gives an ordinary differential equation for $h$

$$
\frac{d h}{d \tau}=\frac{g_{0} L-h}{\tau_{c}}-\frac{P_{\mathrm{in}}(\tau)}{E_{\mathrm{sat}}}\left(e^{h}-1\right) .
$$

Under an appropriate backward injecting power, the inverseoptical-comb (i.e., an inverse Gaussian shape with a small duty cycle in one period) can fully deplete most of the TWSOA gain within one period. This eventually leads to a narrowing effect on the residual gain window of the TWSOA, which remains only 
a Gaussian shape with a small duty cycle. Under a given input pulse shape of $P_{\text {in }}(\tau)$ and gain of $g_{0} L$, if the first term on the right-hand side of (9) is neglected by assuming the pulsewidth $\tau_{p} \ll \tau_{c}$, the solution of (9) is

$$
h(\tau)=-\ln \left\{1-\left(1-\frac{1}{G_{0}}\right) \exp \left[-\frac{U_{\text {in }}(\tau)}{E_{\text {sat }}}\right]\right\}
$$

where $G_{0}=\exp \left(g_{0} L\right)$ is the unsaturated signal-pass amplifier gain, $U_{\text {in }}(\tau)$ is the integral of input power function $P_{\text {in }}(\tau)$ with a form of $U_{\text {in }}(\tau)=\int_{-\infty}^{\tau} P_{\text {in }}(\tau) d \tau$, such that $U_{\text {in }}(\infty)$ will be the input pulse energy. Usually, the instantaneous semiconductor optical amplifier gain is given by

$G(\tau)=\exp [h(\tau)]=\left\{1-\left(1-\frac{1}{G_{0}}\right) \exp \left[-\frac{U_{\text {in }}(\tau)}{E_{\text {sat }}}\right]\right\}^{-1}$.

In contrast to the typical operation of the TWSOA, the rate equation of TWSOA under a serious gain depletion condition with external inverse-optical-comb injection can be greatly simplified. Since the gain window of the TWSOA is temporally modified to implement reamplifying, reshaping, and retiming of the incoming NRZ data stream. Theoretically, the transfer function of the TWSOA transient gain under backward inverseoptical-comb injection can be deduced by modifying the similar rate equation described in [40]. It is necessary to simulate the pulse propagation through the temporally gain-depleted TWSOA with a traveling-wave equation containing spatial and time derivatives. In our case, the backward inverse-optical-comb injection function exhibits a form of $P_{\text {in }}(\tau)=P_{0}\left[1-P^{\prime}(\tau)\right]$ and a Gaussian pulse shape of $P^{\prime}(\tau)$. If we assume an inverseoptical-comb injection to deplete most of TWSOA gain within one period and to leave a narrow gain-window with a Gaussian shape in time domain, the waveform of the input inverse-opticalcomb can be described as

$$
P_{\text {in }}(\tau)=\frac{E_{\text {in }}}{\tau_{0} \sqrt{\pi}}\left[1-\exp \left(-\frac{\tau^{2}}{\tau_{0}^{2}}\right)\right]
$$

where $\tau_{0}$ is the inverse-optical-comb pulsewidth, and the first term on right-hand side is used to completely deplete the TWSOA gain by inducing stimulated emission with the inverseoptical-comb at longer injecting wavelength, and the second term leaves the residual gain window of the TWSOA with a very short duty cycle in one period. Such a function precisely describes the power waveform of a backward injected inverseoptical-comb before entering the TWSOA. With the backward injection, the original TWSOA gain can almost be depleted in one period except a narrow window. By substituting the inverseoptical-comb into the rate equation, we have

$$
\begin{aligned}
\frac{d h}{d \tau}= & \frac{g_{0} L-h}{\tau_{c}}-\frac{P_{\text {in }}(\tau)}{E_{\text {sat }}}=\frac{g_{0} L-h}{\tau_{c}} \\
& -\frac{\left(E_{\text {in }} / \tau_{0} \sqrt{\pi}\right)\left[1-\exp \left(-\tau^{2} / \tau_{0}^{2}\right)\right]}{E_{\text {sat }}} \\
= & \left(\frac{g_{0} L}{\tau_{c}}-\frac{E_{\text {in }}}{\tau_{0} \sqrt{\pi} E_{\mathrm{sat}}}\right)+\frac{E_{\text {in }}}{\tau_{0} \sqrt{\pi} E_{\mathrm{sat}}} \exp \left(-\frac{\tau^{2}}{\tau_{0}^{2}}\right)-\frac{h}{\tau_{c}} .
\end{aligned}
$$

To simplify the previous rate equation, the first term at the right-hand side should be completely subtracted to zero except the gain with a Gaussian shape and a decaying term $h / \tau_{c}$ caused by the recombination induced gain attenuation. In our case, we assume that the decaying time for carriers is comparable with the ultrashort comb pulsewidth under the intense injection of the backward inverse-optical-comb. The residual gain of TWSOA thus becomes an inverse shape of the injected inverse-opticalcomb waveform. The maximum backward injection energy is set as $P_{\text {in }}=E_{\text {in }} / \tau_{0 \pi}^{-1 / 2} \approx\left[g_{0} L / \tau_{c}\right] E_{\text {sat }}$ to completely deplete the gain of the TWSOA in our case. Therefore, we can simplify and rewrite (13) as

$$
\frac{d h}{d \tau}=\frac{g_{0} L}{\tau_{c}} \exp \left(-\frac{\tau^{2}}{\tau_{0}^{2}}\right)-\frac{h}{\tau_{c}}
$$

and the solution of (14) can be solved and rewritten as

$$
\begin{aligned}
h & =e^{-\int f(\tau) d \tau}\left[\int e^{\int f(\tau) d \tau} \frac{g_{0} L}{\tau_{c}} e^{-\tau^{2} / \tau_{0}^{2}} d \tau\right] \\
& =e^{-\tau / \tau_{c}} \frac{g_{0} L}{\tau_{c}} \int e^{\tau / \tau_{c}} e^{-\tau^{2} / \tau_{0}^{2}} d \tau \\
& =-\frac{\tau}{\tau_{c}} \frac{g_{0} L}{\tau_{c}}\left[\frac{-\sqrt{\pi} \tau_{0} e^{\tau_{0}^{2} / 4 \tau_{c}^{2}}}{2} \operatorname{erf}\left(\frac{\tau_{0}}{2 \tau_{c}}-\frac{\tau}{\tau_{0}}\right)\right] \\
& =-\frac{g_{0} L \tau_{0} \sqrt{\pi}}{2 \tau_{c}} e^{\left(\tau_{0}^{2} / 4 \tau_{c}^{2}\right)-\left(\tau / \tau_{c}\right)} \operatorname{erf}\left(\frac{\tau_{0}}{2 \tau_{c}}-\frac{\tau}{\tau_{0}}\right)
\end{aligned}
$$

where erf denotes the error function. The on-level of the input inverse-optical-comb power function first reduces the TWSOA gain to below transparent (i.e., loss) condition. Subsequently, the TWSOA gain build up again within the instantaneous off-level of the inverse-optical-comb function, providing a short rising edge and a carrier-lifetime limited trailing-edge small-signal gain function. Such an operation eventually reshapes the $\mathrm{CW}$ gain of the TWSOA into a greatly shortened gain window. The transient gain windows of the TWSOA are strongly confined by the input inverse-optical-comb pulsewidth, in which larger pulsewidth inevitably results in broaden gain window. That is, the pulsewidth of the converted RZ signal is mainly affected by two parameters, i.e., the pulsewidth of the inverse-opticalcomb and the lifetime of carriers in TWSOA. However, the inverse-optical-comb pulsewidth will play important role on the converted RZ pulsewidth when its injection power is increased to a relatively high level. Similar result for the data pulsewidth shortening effect under strong holding beam injection has also been reported and explained with a theoretical model previously [45]. The injected inverse-optical-combs of two different fullwidth at half-maximum (FWHM), and the simulating results $G(\tau)$ of the TWSOA with the following format are shown in Fig. 4(a) and (b), respectively

$$
\begin{aligned}
G(\tau) & =\exp [h(\tau)] \\
& =\exp \left\{-\frac{g_{0} L \tau_{0} \sqrt{\pi}}{2 \tau_{c}} e^{\left(\tau_{0}^{2} / 4 \tau_{c}^{2}\right)-\left(\tau / \tau_{c}\right)} \operatorname{erf}\left(\frac{\tau_{0}}{2 \tau_{c}}-\frac{\tau}{\tau_{0}}\right)\right\} .
\end{aligned}
$$




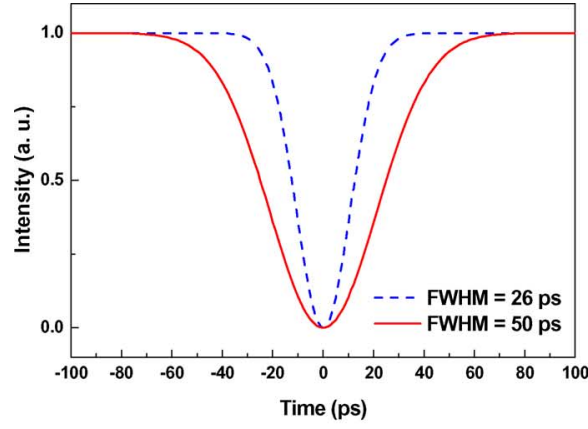

(a)

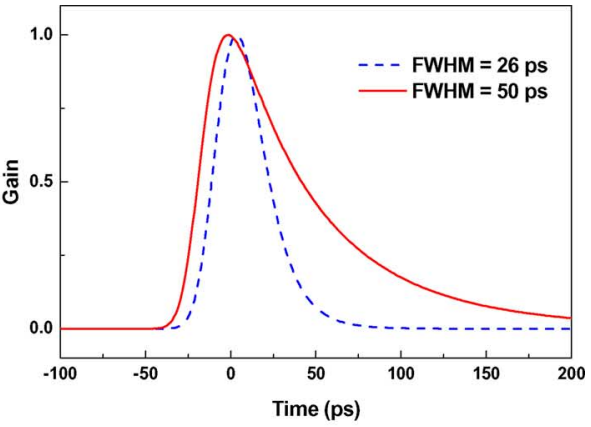

(b)

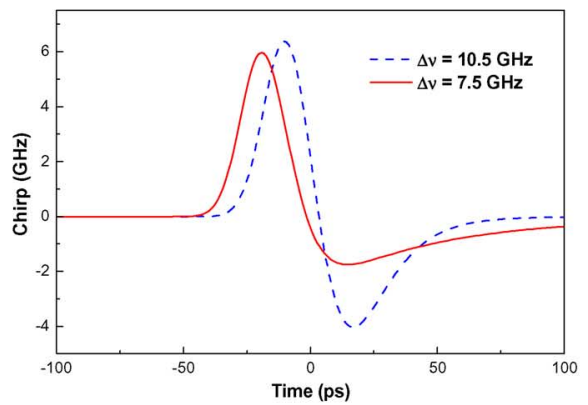

(c)

Fig. 4. (a) Simulated shape of an inverse-optical-comb signal within one period. (b) Simulation of transient gain $[G(\tau)]$ in the TWSOA. (c) Simulation of the dynamic frequency chirp of the converted RZ signals under inverse-optical-comb injection.

In our simulation of 16, we normalized the TWSOA gain and set the gain recovery time and carrier lifetime of the TWSOA as 200 and 25 ps, respectively [46]-[48]. The gain recovery time is quoted from references elsewhere [49]. In previous work, it was found that the gain recovery time can be faster when the data wavelength is shorter that the inverse-optical-comb wavelength. The smallest of the effective carrier recovery time during strong injection condition can be as short as 25 ps [45]. Since the TWSOA is operated at seriously gain-depleted condition, both the effects of gain-recovery time and carrier lifetime to the gain shape of the TWSOA are greatly suppressed. The converted $\mathrm{RZ}$ shape is mainly decided by the inverse-optical-comb itself when gain recovery process of TWSOA is accelerated by strong inverse-optical-comb injection [45], [48].

In addition, the dynamic frequency chirp $(\Delta \omega=-d \phi / d \tau)$ imposed on the output of TWSOA can be derived from the phase modulation, which is proportional to the carrier-induced transient variation on refractive index and gain of the TWSOA (i.e., $\Delta v=\alpha_{e}\left[\Gamma v_{g} a\left(n-n_{0}\right)-1 / \tau_{g}\right] / 4 \pi=\alpha_{e}[d P(\tau) / d \tau-$ $\left.R_{\mathrm{sp}}\right] / 4 \pi P_{0}$ [50], [51]. In our case, the chirp of the RZ data stream added by the TWSOA under the backward inverseoptical-comb injection is

$$
\begin{aligned}
\Delta \nu= & \frac{\alpha_{e}}{4 \pi} \exp \left[-\frac{g_{0} L \tau_{0} \sqrt{\pi}}{2 \tau_{c}} e^{\left(\tau_{0}^{2} / 4 \tau_{c}^{2}\right)-\left(\tau / \tau_{c}\right)} \operatorname{erf}\left(\frac{\tau_{0}}{2 \tau_{c}}-\frac{\tau}{\tau_{0}}\right)\right] \\
& \times\left[\frac{g_{0} L \tau_{0} \sqrt{\pi} e^{\left(\tau_{0}^{2} / 4 \tau_{c}^{2}\right)-\left(\tau / \tau_{c}\right)} \operatorname{erf}\left(\left(\tau_{0} / 2 \tau_{c}\right)-\left(\tau / \tau_{0}\right)\right)}{2 \tau_{c}^{2}}\right. \\
& \left.+\frac{g_{0} L e^{\left(\tau_{0}^{2} / 4 \tau_{c}^{2}\right)-\left(\tau / \tau_{c}\right)-\left(\tau_{0} / 2 \tau_{c}-\tau / \tau_{0}\right)^{2}}}{\tau_{c}}\right]
\end{aligned}
$$

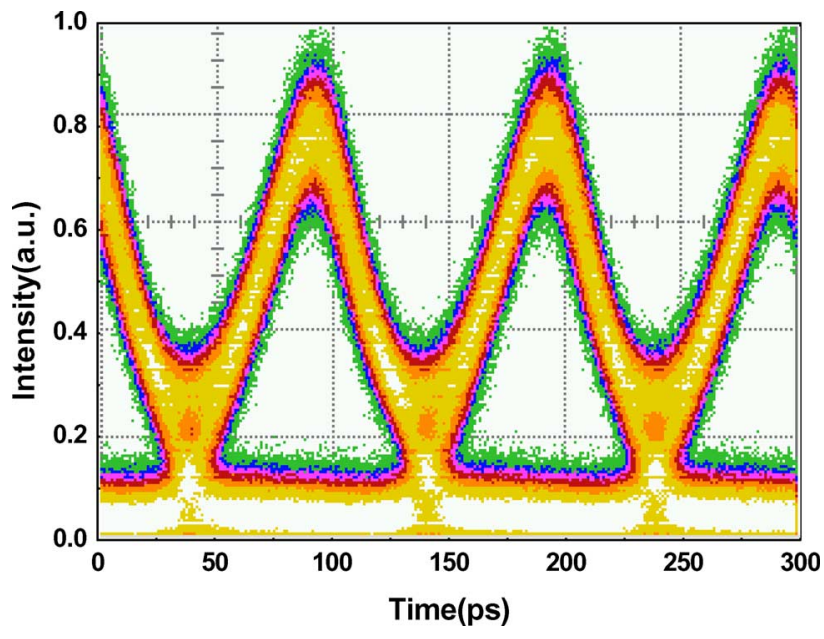

Fig. 5. Eye diagram of the NRZ-to-RZ converted data stream at $10 \mathrm{~Gb} / \mathrm{s}$.

The corresponding chirps of the converted RZ data pulses obtained from the simulating results $G(\tau)$ of the TWSOA with the formats illustrated in Fig. 4(b) are shown in Fig. 4(c).

\section{B. Extinction Ratio and Noise Level}

In previous approach with a DFB LD based backward inverseoptical-comb injection, the NRZ is perfectly reshaped into RZ format (see Fig. 5), and the NRZ-to-RZ data format conversion is achieved only when the inverse-optical-comb injected power is increased beyond a conversion threshold. The converted RZ PRBS exhibits an ON/OFF extinction ratio of $15.5 \mathrm{~dB}$, which is $3.5 \mathrm{~dB}$ higher than the original NRZ PRBS, as illustrated in 


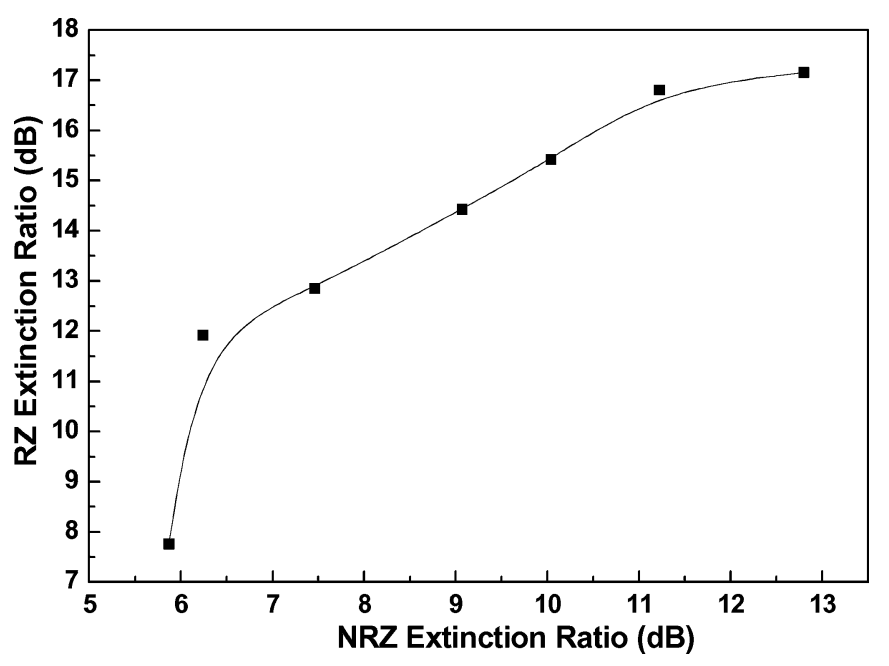

Fig. 6. ON/OFF extinction ratio of the converted RZ data stream versus that of the incoming NRZ data stream.

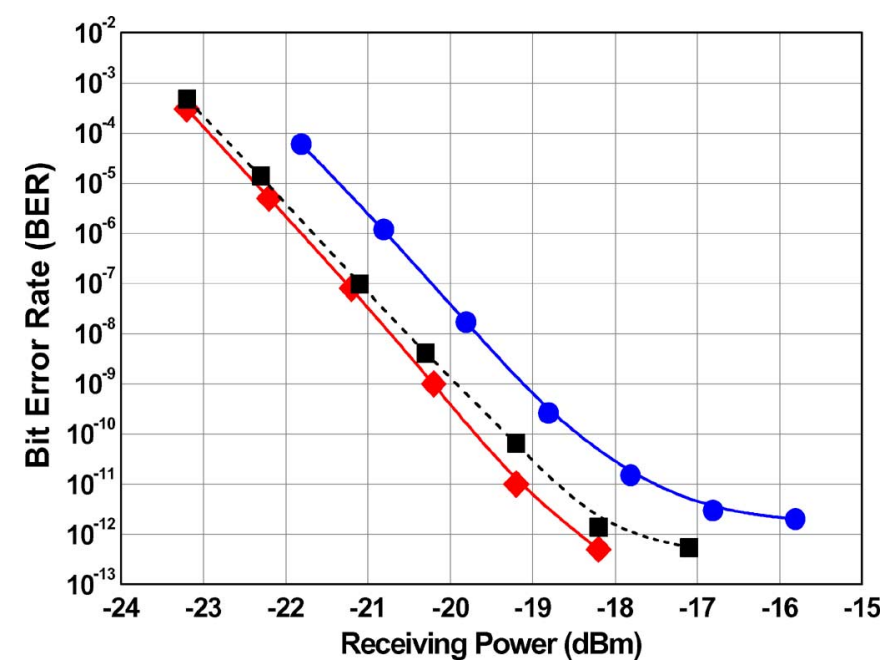

Fig. 7. BER performance of the back-to-back NRZ (circle) and the TWSOA converted RZ under DFBLD (square) and FPLD (diamond) based inverseoptical-comb injection.

Fig. 6. As shown in Fig. 7, the requested (BER) of $10^{-12}$ for data communication is achieved at receiving power as small as $-18.5 \mathrm{dBm}$, providing a negative power penalty of $1.5 \mathrm{~dB}$ as compared to the back-to-back transmitted NRZ condition [52], [53]. The maximum $Q$-factor of the converted RZ is 7.72, corresponding to a reachable BER of $5.8 \times 10^{-15}$. With DFBLD based inverse-optical-comb injection, a negative power penalty of for the BER of the converted RZ data stream is observed. In comparison with the DFBLD case, the FPLD based inverseoptical-comb injection exhibits better BER performance with larger negative power penalty at larger receiving power condition. In principle, a single-mode injection only trigger the stimulated emission of conduction-band carriers at energy states with wavelength shorter than the injection one, which thus consumes carriers upon higher energy levels $\left(E>E_{1}\right)$ and leaves other carriers below the injecting band undepleted in TWSOA. The residual low-energy carriers account for the amplified spontaneous emission of TWSOA at "OFF" state of the NRZ data,

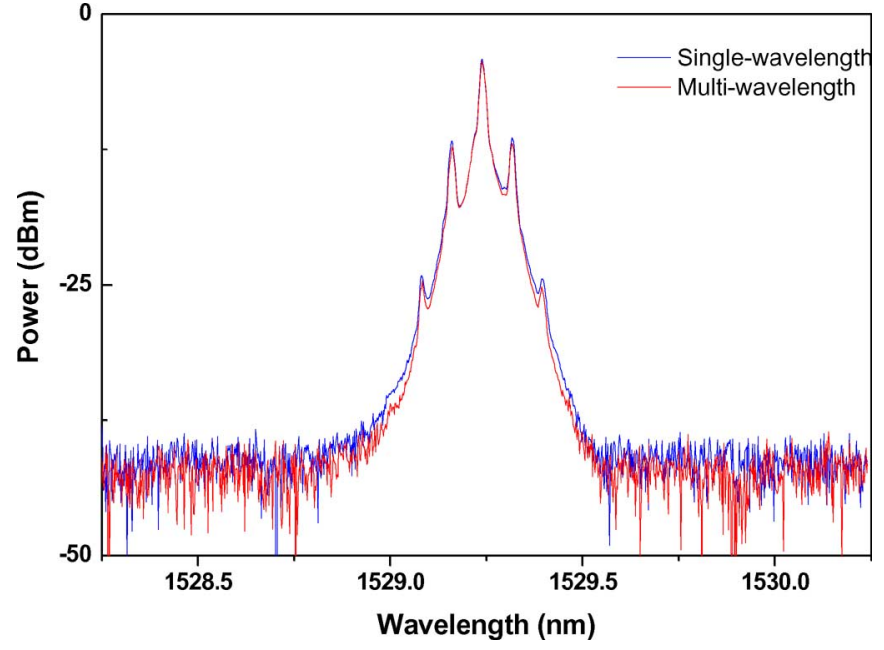

Fig. 8. Optical spectra of the converted RZ data streams obtained from TWSOA under single- (dark gray) and multimode (light gray) inverse-opticalcomb injection.

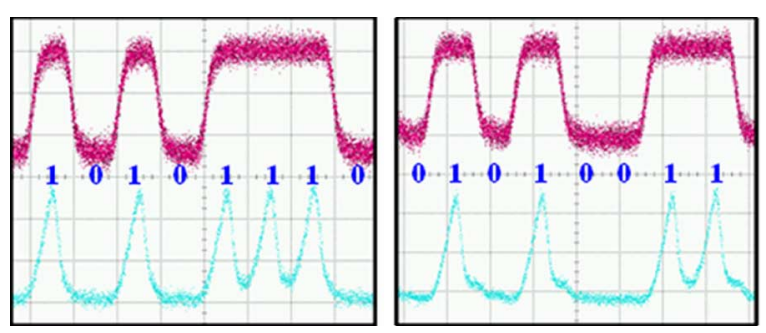

Fig. 9. Left: the incoming NRZ data stream (upper) and its converted RZ data stream (lower) from single-mode inverse-optical-comb injected TWSOA. Right: the incoming NRZ data stream (upper) and its converted RZ data stream (lower) from multi-mode inverse-optical-comb injected TWSOA.

contributing to a bad SNR under NRZ-to-RZ data format conversion, as illustrated in the following figures. If we employ a multimode inverse-optical-comb injection with same power level, which can benefit from the full consumption of whole carriers at conduction band of the TWSOA. Since the multimode FPLD has a relatively broadband lasing spectrum with many long-wavelength modes, which essentially cover the lower energy levels $\left(E<E_{2}\right)$ and entirely depletes conduction-band carriers in the TWSOA, the depleted carrier concentration $\Delta N_{1}$ obtained under DFBLD injection is far smaller than $\Delta N_{2}$ obtained under FPLD injection. Both the RZ spectra obtained under the FPLD and DFBLD based inverse-optical-comb injections are shown in Fig. 8. We compare two spectra and conclude that the multimode FPLD injection provides a better optical SNR than the single-mode DFBLD does. The other supporting evidence is the improved ON/OFF extinction ratio of the converted RZ data stream, which can be seen from Fig. 9 that illustrates the temporal trace of the converted RZ data stream with the TWSOA under single- or multimode optical injection. The left part of Fig. 9 shows a slightly large noise level at off bits under single-mode dark-optical-comb injection condition due to the unwanted amplified spontaneous emission induced by residual carriers in TWSOA. In comparison, the multimode injection case in right figure reveals a small fluctuation (even not so discriminated), and thus, give rise to a better ON/OFF 

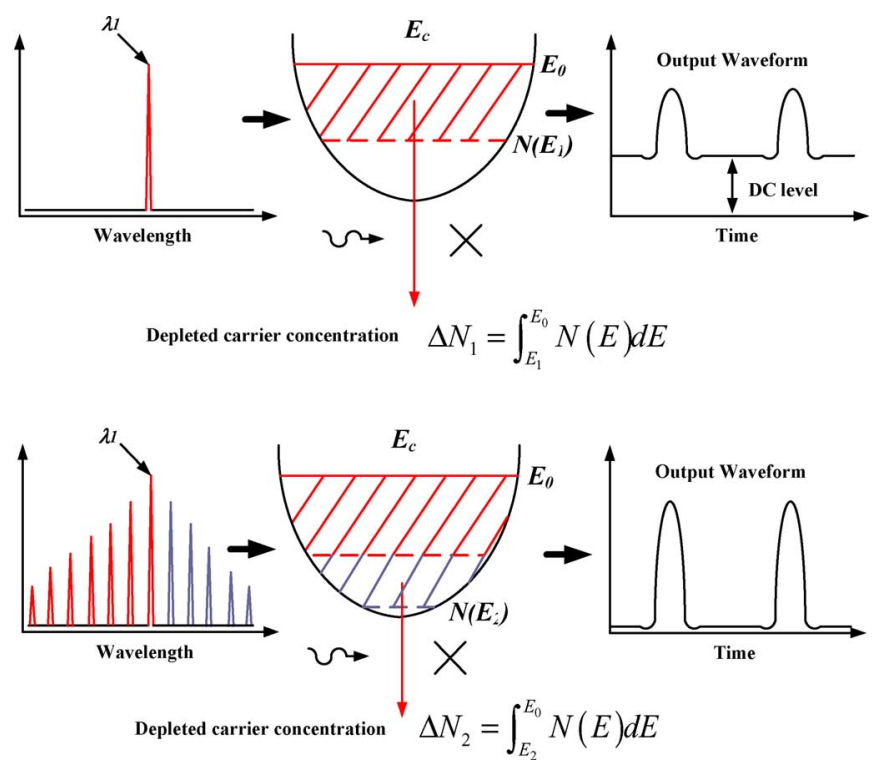

Fig. 10. Scheme of the carrier depletion in the TWSOA under single- (upper part) and multimode (lower part) inverse-optical-comb injection.

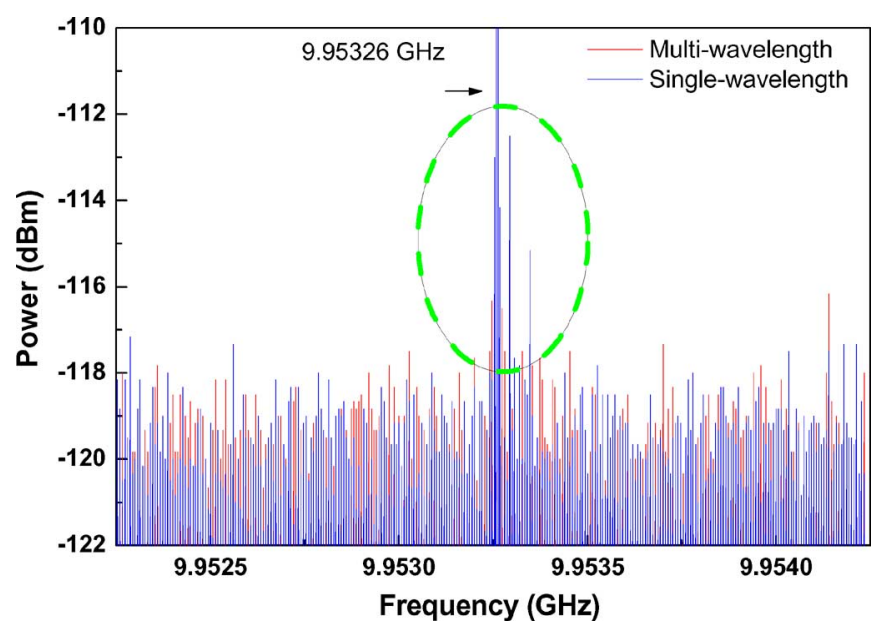

Fig. 11. Comparison on the RF spectra of the RZ data-streams obtained from DFBLD (dark gray) and FPLD (light gray) injected TWSOAs.

extinction ratio, since there are no residual carriers with such a short NRZ-to-RZ conversion window to contribute amplifiedspontaneous emission (ASE) noise in this case. More carriers left in the conduction band will contribute to a higher noise level due to the larger spontaneous emission, and also provide a limited ON/OFF extinction ratio due to an higher upward offset of the " 0 " level in the converted RZ data stream, as shown in Fig. 10. The gain of the TWSOA cannot be fully depleted if the inverse-optical-comb injects into the TWSOA at wavelength nearly the original gain peak (i.e., backward injection at shorter wavelength).

As expected, a multimode inverse-optical-comb injection with same power level benefits from the complete depletion of whole carriers in whole conduction band of the TWSOA. Therefore, the noise level of the RZ data stream from the FPLD injected TWSOA is at least $1 \mathrm{~dB}$ lower than that from a DFBLD injected TWSOA. Such a difference in noise power between the single- and multimode inverse-optical-comb injections is measured and shown in Fig. 11, the related RF frequency spectra by using a spectrum analyzer with resolution bandwidth $(\mathrm{RBW})=$ $10 \mathrm{~Hz}$, video bandwidth $(\mathrm{VBW})=10 \mathrm{~Hz}$, span $=2 \mathrm{MHz}$ (Hewlett Packard, HP8565E). It is significant that the side-peak noise level of a RZ data signal obtained from a single-mode DFBLD injected TWSOA is larger than that obtained from a multimode FPLD injected TWSOA. These results clearly interpret a better noise performance of the NRZ-to-RZ converted data stream generated by the TWSOA under multimode injection. With FPLD based inverse-optical-comb injection, the converted RZ also shows an ON/OFF extinction of better than $17 \mathrm{~dB}$, which is already $1 \mathrm{~dB}$ larger than the DFBLD injection case. In a static measurement, the ultimate ON/OFF extinction ratio of the wavelength-converted signal in a traditional XGM-controlled TWSOA can be determined as up to $30 \mathrm{~dB}$ by the transfer function of output power against input power. However, the dynamic ON/OFF extinction ratio of the wavelength-converted NRZ signal is always degraded than a theoretically estimated value of $20 \mathrm{~dB}$. There are two mechanisms responsible for such degradation. First, the TWSOA carrier number can always reach a steady-state value in the static (or $\mathrm{CW}$ ) regime, leading to a small output at "0" level. However, in dynamic operation, the TWSOA carrier number is unable to reach this steady-state value due to the finite carrier lifetime, hence, the output at " 0 " level raises to be not negligible. Second, the noises of the optical receiver and the oscilloscope system set a detecting limit for the converted RZ data stream with a finite extinction ratio. Technically, the improvement on the extinction ratio of RZ data can be achieved by applying a higher current or using larger injecting power to the TWSOA for reducing carrier lifetime. The TWSOA output power versus the inverse-optical-comb input power is shown in Fig. 12. Our proposed scheme is slightly different from the traditional wavelength conversion scheme achieved by the TWSOA with XGM, in which the ultimate ON/OFF extinction ratio of the converted RZ signal (see right part of Fig. 12) is unable to be determined by the transfer function of the output power versus input power in the static state, since the gain of the TWSOA is seriously deficient within a period due to a high-power backward inverse-optical-comb injection. On the other hand, the sensitivity of the sampling oscilloscope used in this experiment may be in sufficient for diagnosis of the converted RZ signal. In a practical fiber-optic communication system, all measurements for the RZ or NRZ signals (i.e., extinction ratio, SNR, BER, jitter, etc.) must be processed after receiving. Thus, the signal performances are eventually limited by the bandwidth and sensitivity of the receiver and the oscilloscope even if they have a better quality intrinsically. By setting two inverse-optical-combs with FWHM of 50 and $26 \mathrm{ps,}$ respectively, our simulations reveal that the lengthening tail of TWSOA gain in time domain also degrades the ON/OFF extinction ratio of the converted RZ data bit, as shown in Fig. 13. The calculation elucidates that a short-pulsewidth inverseoptical-comb injection remains a narrowest gain window in TWSOA, thus providing shorter falling time and better ON/OFF extinction for the output data bit during NRZ-to-RZ format conversion. 


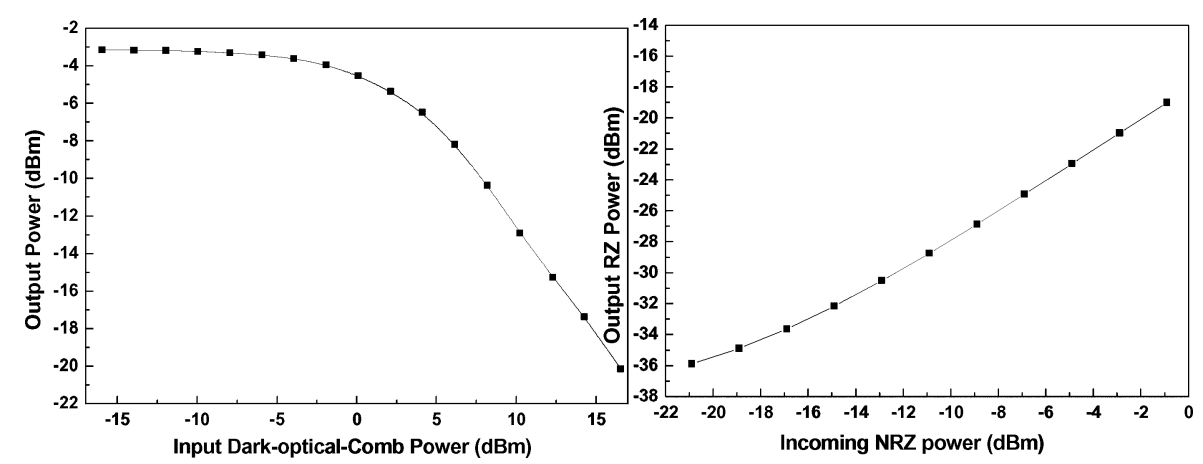

Fig. 12. Left: output power of TWSOA versus inverse-optical-comb power. Right: output power of TWSOA versus input NRZ power.

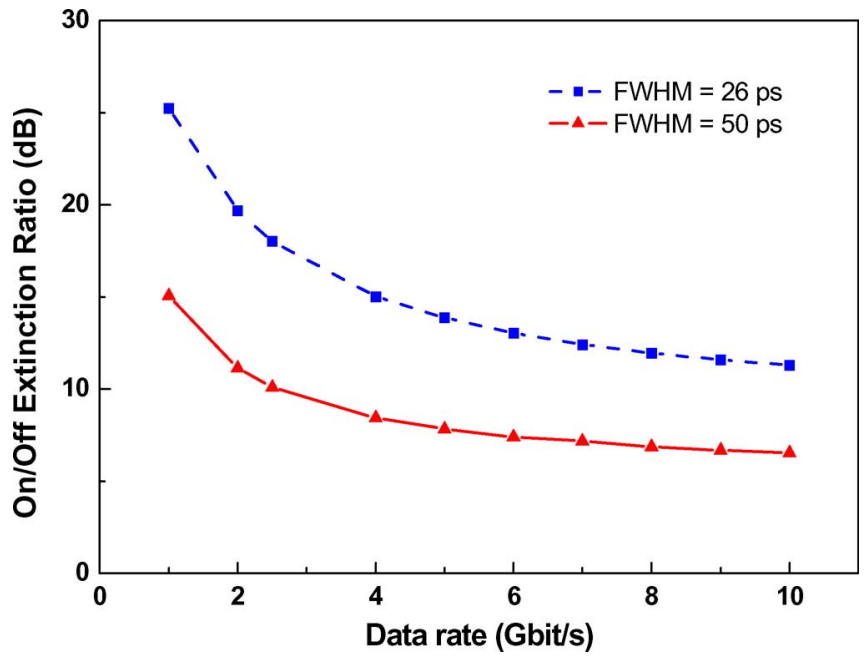

Fig. 13. Calculated ON/OFF extinction ratio versus data rate with different width gain window.

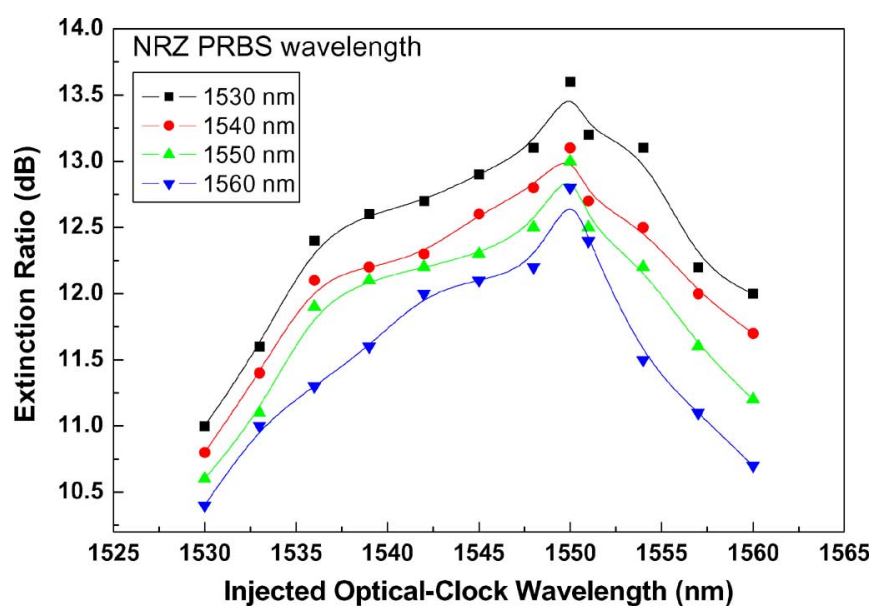

Fig. 14. ON/OFF extinction ratio as a function of the injected inverse-opticalcomb wavelength.

\section{Pulsewidth and Frequency Chirp}

In our proposed architecture, the preserved RZ data conversion means that the SOA-based NRZ-to-RZ data format converter can transform the incoming optical NRZ stream into the RZ data stream without changing its wavelength. The wave-

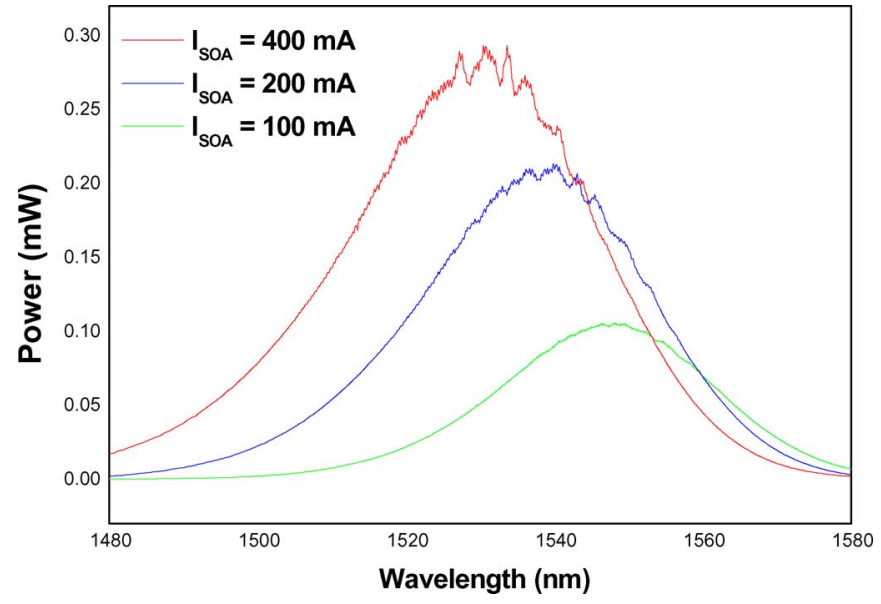

Fig. 15. Gain spectrum of TWSOA at different bias currents.

length of data stream is thus preserved during the data conversion process. The only difference is that the inverse-opticalcomb injected at a wavelength longer than that of the input NRZ data stream. As shown Fig. 14, the ON/OFF extinction ratio of the converted RZ PRBS data stream is dependent on the wavelength of the backward inverse-optical-comb injection, which reveals the largest ON/OFF extinction ratio with backward inverse-optical-comb injection at 1550-1555 nm when the NRZ PRBS data are set at shorter wavelengths. Indeed, for a determined NRZ wavelength at $1530 \mathrm{~nm}$, the extinction ratio of the converted RZ signal will reach its maximum at an inverse-optical-comb wavelength around $1550 \mathrm{~nm}$. Afterward, the extinction value will slowly reduce when the operation wavelength of NRZ signal is increased from 1550 to $1560 \mathrm{~nm}$. This is mainly due to the abruptly shifted gain profile of the TWSOA toward longer wavelength under the intense backward injection of inverse-optical-comb. That is the reason why we claim the NRZ PRBS should be set at the vicinity of gain peak of TWSOA. Originally, the gain profile of TWSOA is centered at $1550 \mathrm{~nm}$. At highly dc-biased condition, the gain profile of TWSOA slightly blue shifts to $1530 \mathrm{~nm}$, as shown in Fig. 15. Therefore, the NRZ PRBS is setting at the vicinity of gain peak of TWSOA. The blue shift of TWSOA gain profile toward shorter wavelength is a result of band filling effect. In Fig. 16, it is clearly illustrated that the gain profile of the transparent TWSOA (strongly 


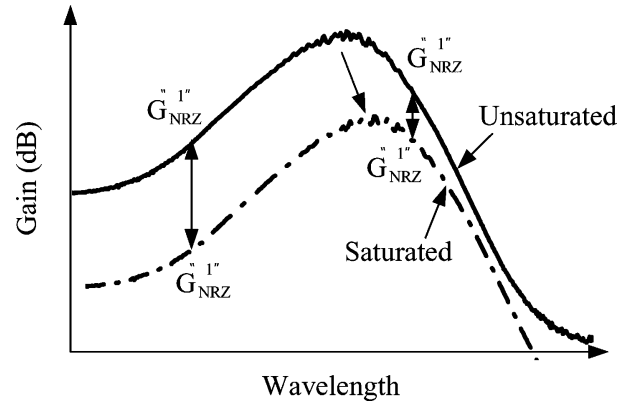

Fig. 16. Illustration on the enhancement of ON/OFF extinction ratio by red shifting TWSOA gain profile back to its transparent condition under strong injection of inverse-optical-comb.

depleted by inverse-optical-comb) will be red shifted as compared to that of the TWSOA at highly biased condition (without inverse-optical-comb injection). Alternatively, it is the recovery of TWSOA gain back to its transparent (zero bias) condition. By operating the TWSOA gain between these two conditions, the maximum ON/OFF extinction is achieved when the inversionoptical-comb injecting wavelength is coincident with the band gap of the TWSOA at nearly transparent condition. Such a gaindepletion induced red shift of gain profile occurs outside the NRZ-to-RZ conversion window in time domain, which essentially results in a largest extinction ratio for the input NRZ and converted RZ signals at a shorter wavelength. Detuning the inverse-optical-comb to longer wavelength will make the photon energy below the band gap energy of TWSOA, thus fail to deplete the TWSOA gain, and shift its gain profile for enlarging the ON/OFF extinction ratio of the converted RZ signal. The input and output data streams remain identical wavelength during the NRZ-to-RZ conversion. This is the most intriguing feature of our proposed scheme as compared to previous works. For optical time-division-multiplexing (OTDM) application, it is requisite to generate an RZ data bit with shorter duty cycle for increasing the channel numbers and communication capacity. In principle, the limitation on the output pulsewidth of the TWSOA-based RZ pulsed data converter is mainly determined by its effective carrier lifetime of $\tau=\left[\tau_{s}^{-1}+d\left(g P_{\text {in }}\right) / d n\right]^{-1}$ [40]. It interprets the rise/fall time as well as the duty cycle of the converted RZ data bit can essentially be shortened due to the decreasing carrier lifetime in a highly biased TWSOA with strong optical injection, as shown in Fig. 17. With single and multimode inverse-opticalcomb injection, the converted RZ pulsewidths are shortened from 37 to $31 \mathrm{ps}$ and from 42 to $32 \mathrm{ps,}$, respectively, as the injection power enlarges from 2.4 to $16.5 \mathrm{dBm}$. The observed rising time of the converted RZ pulse remains almost unchanged; however, the falling time was monotonically reduced by increasing the injection power of inverse-optical-comb. The evolution on pulsewidth of the converted RZ data with increasing injection power exhibits similar decreasing trend with its falling time. To provide a fast conversion speed and shortened response, the increase in both the biased current of the TWSOA and the injection power of inverse-optical-comb are mandatory. In comparison with the single-mode inverse-optical-comb injection, the multimode inverse-optical-comb injection induces a RZ data slightly lengthened rise/fall time and broaden pulsewidth under low-power injection case. At same injecting power, such an FWHM lengthening effect is mainly attributed to the lower power spectral density, $P(\lambda)$, of the multimode inverse-opticalcomb as compared to that of the single-mode one, which is insufficient to deplete the carriers as well as the gain of the highly-biased TWSOA in whole spectral region. By further reducing the biased current or increasing the injecting power, the gain depletion becomes serious such that the switching time of the TWSOA under single- and multimode inverse-optical-comb injection conditions can be almost identical.

The dynamic frequency chirp of the TWSOA-converted RZ data can also be simulated and measured, however, which reveal a linearly increasing trend with the enlarged inverse-opticalcomb injection power. Under multimode inverse-optical-comb injection, the peak frequency chirp of the pulsed RZ data is concurrently enlarged from 2 to $5 \mathrm{GHz}$ as the inverse-opticalcomb power increases from 2.4 to $16.5 \mathrm{dBm}$, as shown in Fig. 17(d). In comparison with single-mode injection induced chirp of 4-8 GHz, the multimode inverse-optical-comb injection leads to a smaller frequency chirp due to its broader pulsewidth. Nevertheless, the increasing trend of the frequency chirp with inverse-optical-comb power exhibits a slope of $0.3 \mathrm{GHz} / \mathrm{dB}$. Obviously, the single-mode inverse-optical-comb injection shortens the converted RZ pulsewidth at a cost of enlarged dynamic frequency chirp, whereas the multimode inverse-optical-comb injection only contributes a rising, but saturable peak-to-peak frequency chirp up to $5 \mathrm{GHz}$. Such a large frequency chirp is proportional to the XGM depth, which can be minimized if we concurrently reduce the TWSOA biased current and shrink its gain bandwidth, together with temporally and spectrally gain slicing by multimode inverse-optical-comb injection. The decreasing peak-to-peak chirp of as small as $1.2 \mathrm{GHz}$ is obtained by reducing TWSOA current to $100 \mathrm{~mA}$, as shown in Fig. 18. Our results conclude that the multimode inverse-optical-comb can provide better performance than single-mode one on reducing the dynamic frequency chirp at lower injection power and biased current condition. Both the gain and the spectral linewidth of the TWSOA are simultaneously minimized in this case.

Typical operation of NRZ-to-RZ conversion is implemented by using optical-electrical-optical (OEO) or optical-optical (or all-optical) approaches. Significantly, the OEO demonstration is somewhat limited by the finite bandwidth of the electronic NRZto-RZ IC (typically at $12.5 \mathrm{~Gb} / \mathrm{s}$ ). In contrast, the optical NRZ injection induced RZ data conversion in a gain-switched FPLD or a gain-reshaped TWSOA via XGM technique are currently two of the intriguing approaches. However, both techniques are restricted by the finite electrical modulation bandwidth of the LD and SOA. To overcome, the external inverse-opticalcomb modulation architecture has inevitably been employed in our scheme. Our current approach is to transfer the bandwidth bottleneck problem from the TWSOA to the RF-amplifierdriven electrical comb and external modulator. Because it is relatively easy to obtain a wide-band microwave comb generator and amplifier instead of finding an LD or TWSOA with greatly improved electrical modulation performance. Under the 


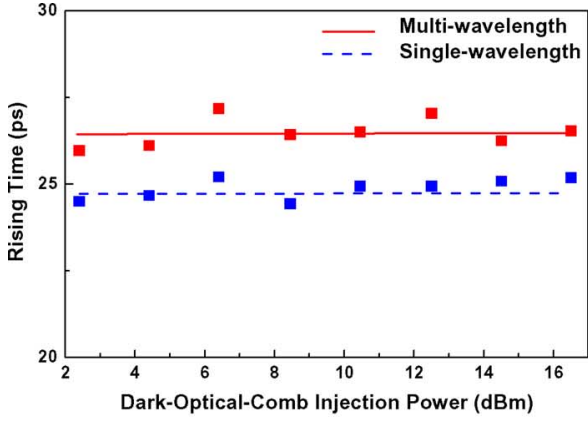

(a)

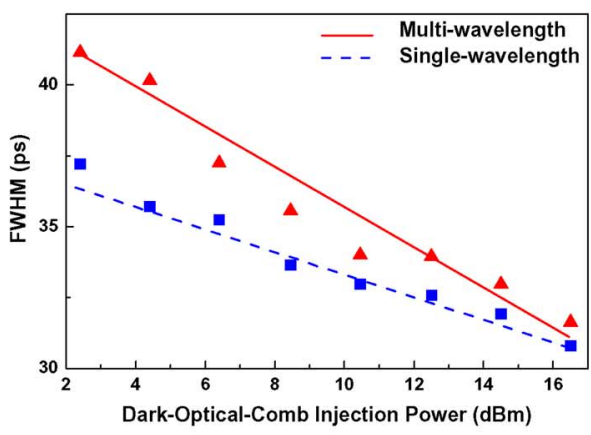

(c)

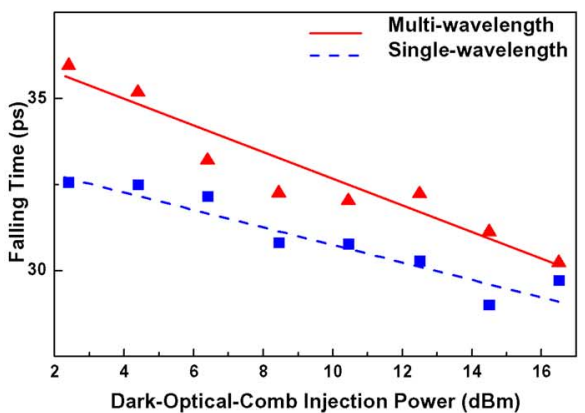

(b)

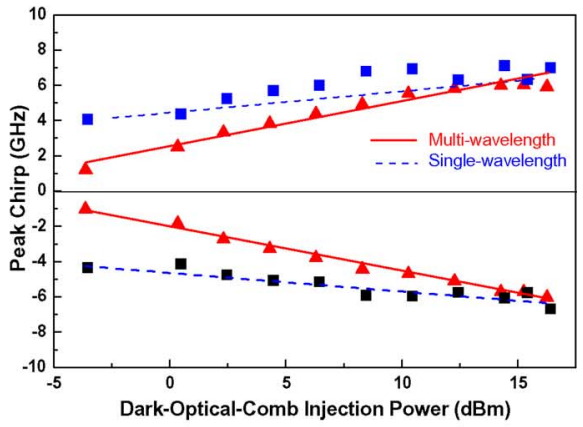

(d)

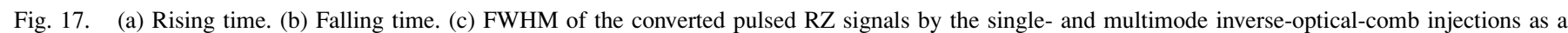

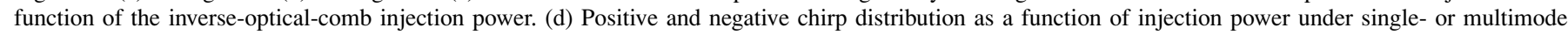
inverse-optical-comb injection.

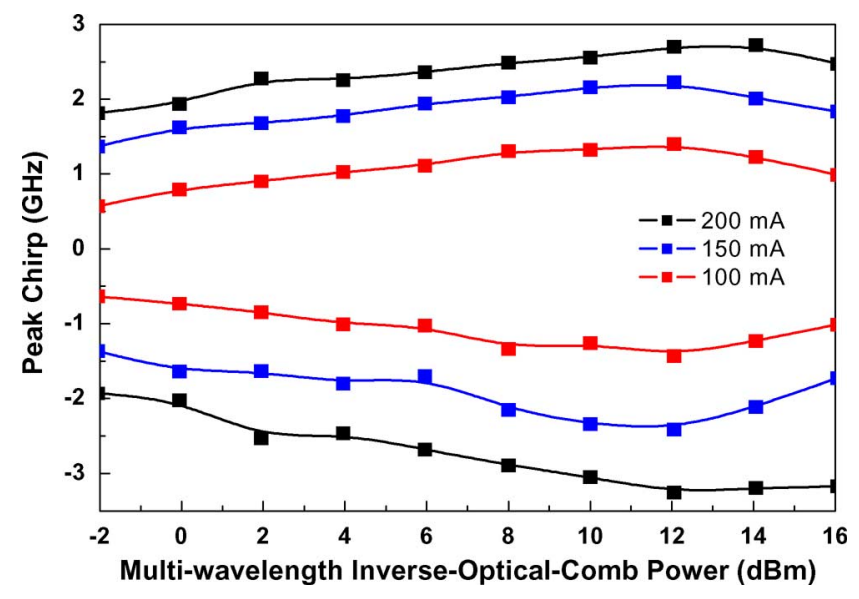

Fig. 18. Dependence of TWSOA-biased current and the positive and negative chirp distribution as a function of multimode inverse-optical-comb injection power.

injection of a multiwavelength inverse-optical-comb pulse train, the overlapped wavelength region of the broadband gain profile of TWSOA and the inverse-optical-comb will simultaneously be depleted each other to leave a reshaped narrow gain spectrum. In practical application, there are two possible candidates for generating broadband inverse-optical-comb signal in our system. One is the broadband ASE and the other is the FPLD. Indeed, the multimode partition noise associated with the multiwavelength inverse-optical-comb generated from the FPLD could be transferred into the converted signal, which is also an important issue to be improved in the near future. However, the use of a broadband ASE-based inverse-optical-comb as the injection source also introduces large relative intensity noise, and inevitably degrades the transmission as well as the BER performance of the converted RZ data. The proposed scheme can definitely be used for NRZ-to-RZ conversion at bit rates higher than $10 \mathrm{~Gb} / \mathrm{s}$ with the availability of high-frequency electrical comb based inverse-optical-comb generator. In comparison with other proposed techniques, our approach is relatively simplified without any specific fiber-optic elements except a microwave amplifier for driving electrical comb generator. The proposed techniques thus can be an alternative method among all of the previously demonstrated schemes.

\section{CONCLUSION}

The comparisons on shortened falling time, reduced chirp, and improved ON/OFF extinction ratio of $10 \mathrm{~Gb} / \mathrm{s}$ all-optical data-format converted pulse stream from single- and multimode inverse-optical-comb injected TWSOA with temporally and spectrally reshaped gain window are demonstrated. The FWHM of the multi- and single-mode injection converted pulsed RZ data are 31.6 and 30.8 ps, respectively. The rising time remain almost unchanged; however, the falling time can be monotonically reduced by increasing injection power. The evolution of FWHM with increasing injection power exhibits similar trend with falling time. The maximum $Q$-factor of the converted RZ is 7.72, corresponding to a reachable BER of $5.82 \times 10^{-15}$. However, the finite falling time restricts the further improvement on the extinction ratio and BER at $17 \mathrm{~dB}$ and $10^{-13}$. The NRZ-to-RZ conversion in the TWSOA under intense 
inverse-optical-comb XGM not only results in patterning, but also induces large chirp with its level proportional to the amount of gain depletion. The converted pulsed RZ signal is subject to the net effect of addition frequency chirp as well as dispersion being to increase the power penalty of the RZ data stream. With a low-biased TWSOA, the peak-to-peak chirp of the converted RZ pulsed data can be as low as $2.7 \mathrm{GHz}$, respectively. Even at high gain operation, the converted RZ data obtained by multimode inverse-optical-comb injection still reveals a peak-topeak frequency chirp of $10 \mathrm{GHz}$ smaller than that of $13.2 \mathrm{GHz}$ obtained under the single-mode inverse-optical-comb injection case. The chirp can abruptly be reduced to $1.2 \mathrm{GHz}$ by reducing the TWSOA-biased current $100 \mathrm{~mA}$.

\section{REFERENCES}

[1] K. Mishina, S. M. Nissanka, A. Maruta, S. Mitani, K. Ishida, K. Shimizu, T. Hatta, and K. I. Kitayama, "All-optical modulation format conversion from NRZ-OOK to RZ-QPSK using parallel TWSOA-MZI OOK/BPSK converters," Opt. Exp., vol. 15, pp. 7774-7785, 2007.

[2] X. Yang, A. K. Mishra, R. J. Manning, R. P. Webb, and A. D. Ellis, "All-optical $42.6 \mathrm{Gbit} / \mathrm{s} \mathrm{NRZ-to-RZ} \mathrm{format} \mathrm{conversion} \mathrm{by} \mathrm{cross-phase}$ modulation in single TWSOA," Electron. Lett., vol. 43, pp. 890-892, 2007.

[3] X. Yang, A. K. Mishra, R. J. Manning, R. P. Webb, and A. D. Ellis, “Alloptical $40 \mathrm{Gbit} / \mathrm{s}$ NRZ-to-RZ format conversion by nonlinear polarisation rotation in TWSOAs," Electron. Lett., vol. 43, pp. 69-471, 2007.

[4] T. G. Silveira, A. Teixeira, G. T. Beleffi, D. Forin, P. Monteiro, H. Furukawa, and N. Wada, "All-optical conversion from RZ to NRZ using gain-clamped TWSOA," IEEE Photon. Technol. Lett., vol. 19, no. 18, pp. 357-359, Sep. 2007.

[5] J. Dong, X. Zhang, J. Xu, D. Huang, S. Fu, and P. Shum, "Theoretical study of TWSOA-based wavelength conversion with NRZ and RZ format at $40 \mathrm{~Gb} / \mathrm{s}$," Chin. Phys. Lett., vol. 24, pp. 990-993, 2007.

[6] J. Dong, X. Zhang, J. Xu, D. Huang, S. Fu, and P. Shum, "40 Gb/s all-optical NRZ-to-RZ format conversion using single TWSOA assisted by optical bandpass filter," Opt. Exp., vol. 15, pp. 2907-2914, 2007.

[7] Y.-C. Chang, Y.-H. Lin, J. Chen, and G.-R. Lin, “All-optical NRZ-to-PRZ format transformer with an injection-locked Fabry-Perot laser diode at unlasing condition," Opt. Exp., vol. 12, pp. 4449-4456, 2004.

[8] K. Mishina, A. Maruta, S. Mitani, T. Miyahara, K. Ishida, K. Shimizu, T. Hatta, K. Motoshima, and K. Kitayama, "NRZ-OOK-to-RZ-BPSK modulation-format conversion using TWSOA-MZI wavelength converter," J. Lightw. Technol., vol. 24, pp. 3751-3758, 2006.

[9] L. Yin, Y. Yan, Y. Zhou, J. Wu, and J. Lin, "Novel scheme for all-optical clock recovery from NRZ signal," Microw. Opt. Technol. Lett., vol. 48, pp. 516-521, 2006.

[10] Y. Yu, X. Zhang, and D. Huang, "All-optical clock recovery from NRZDPSK signal," IEEE Photon. Technol. Lett., vol. 18, no. 22, pp. 23562358, Nov. 2006.

[11] Y. A. Leem, D. C. Kim, E. Sim, S.-B. Kim, H. Ko, K. H. Park, D.-S. Yee, J. O. Oh, S. H. Lee, and M. Y. Jeon, "The characterization of all-optical 3R regeneration based on InP-related semiconductor optical devices," IEEE J. Sel. Top. Quantum Electron., vol. 12, no. 4, pp. 726-735, Jul./Aug. 2006.

[12] J. Slovak, C. Bornholdt, J. Klreissl, S. Bauer, M. Biletzke, M. Schlak, and B. Sartorius, "Bit rate and wavelength transparent all-optical clock recovery scheme for NRZ-coded PRBS signals," IEEE Photon. Technol. Lett., vol. 18, no. 7, pp. 844-846, Apr. 2006.

[13] Y. Yan, L. Yin, Y. Zhou, G. Liu, J. Wu, and J. Lin, "10 Gbit/s alloptical NRZ-to-RZ conversion based on TOAD," Proc. SPIE, vol. 6025, pp. R250-R250, 2006.

[14] H. S. Chung, R. Inohara, K. Nishimura, and M. Usami, "All-optical multiwavelength conversion of $10 \mathrm{Gbit} / \mathrm{s}$ NRZ/RZ signals based on TWSOAMZI for WDM multicasting," Electron. Lett., vol. 41, pp. 432-433, 2005.

[15] C. G. Lee, Y. J. Kim, C. S. Park, H. J. Lee, and C. S. Park, "Experimental demonstration of $10-\mathrm{Gb} / \mathrm{s}$ data format conversion between NRZ and RZ using TWSOA-Loop-Mirror," J. Lightw. Technol., vol. 23, pp. 834-841, 2005.

[16] S. Betti, F. Curti, E. Duca, S. Monterosso, A. Reale, and G. M. Tosi-Beleffi, "Optical clock recovery from 10-Gb/s NRZ signal after propagation on $100 \mathrm{~km}$ of DS, NZD or SR installed fiber," Microw. Opt. Technol. Lett., vol. 44, pp. 264-266, 2005.

[17] S. Betti, C. Bulli, F. Curti, E. Duca, S. Persia, A. Reale, and G. M Tosi-Beleffi, "Optical clock recovery from 10-Gb/s NRZ signal," Microw. Opt. Technol. Lett., vol. 42, pp. 435-437, 2004.

[18] W. Li, M. Chen, Y. Dong, and S. Xie, "Optimization and evaluation of an all-optical NRZ-to-CSRZ format converter using TWSOA-based fiber loop mirror," Proc. SPIE, vol. 5281, pp. 145-149, 2004.

[19] W. Li, M. Chen, Y. Dong, and S. Xie, "All-optical format conversion from NRZ to CSRZ and between RZ and CSRZ using TWSOA-based fiber loop mirror," IEEE Photon. Technol. Lett., vol. 16, no. 1, pp. 203-205, Jan. 2004.

[20] H. Takeda, F. Kondo, and H. Uenohara, "Modulation format dependence of wavelength conversion performance using cross gain modulation in a semiconductor optical amplifier," Jpn. J. Appl. Phys., vol. 42, no. 3A, pp. L252-L254, 2003.

[21] L. X. Wang, B. C. Baby, V. Glesk, and I. Prucnal, "All-optical data format conversion between RZ and NRZ based on a Mach-Zehnder interferometric wavelength converter," IEEE Photon. Technol. Lett., vol. 15, no. 2, pp. 308-310, Feb. 2003.

[22] W. Mao, Y. Li, M. Al-Mumim, and G. Li, "All-optical clock recovery for both RZ and NRZ data," IEEE Photon. Technol. Lett., vol. 14, no. 6, pp. 873-875, Jun. 2002.

[23] A. Reale, P. Lugli, and S. Betti, "Format conversion of optical data using four-wave mixing in semiconductor optical amplifiers," IEEE J. Sel.Top. Quantum Electron., vol. 7, no. 4, pp. 703-709, Jul./Aug. 2001.

[24] S.-G. Park, L. H. Spiekman, M. Eiselt, and J. M. Weisenfeld, "Chirp consequences of all-optical RZ to NRZ conversion using cross-phase modulation in an active semiconductor photonic integrated circuit," IEEE Photon. Technol. Lett., vol. 12, no. 3, pp. 233-235, Mar. 2000.

[25] H. J. Lee, K. Kim, J. Y. Choi, H. G. Kim, and C. H. Yim, "All-optical NRZ-to-inverted-RZ converter with extinction ratio enhancement using a modified terahertz optical asymmetric demultiplexer," IEICE Trans. Commun., vol. E82-B, pp. 387-389, 1999.

[26] H. J. Lee, H. G. Kim, J. Y. Choi, and H. K. Lee, “All-optical clock recovery from NRZ data with simple NRZ-to-PRZ converter based on self-phase modulation of semiconductor optical amplifier," Electron. Lett., vol. 35, pp. 989-990, 1999.

[27] M. Asghari, I. H. White, and R. V. Penty, "Wavelength conversion using semiconductor optical amplifier," J. Lightw. Technol., vol. 15, pp. 11811190, 1997.

[28] D. Norte and A. E. Willner, "Experimental demonstrations of all-optical conversion between RZ and NRZ data formats incorporating noninverting wavelength shifting leading to format transparency," IEEE Photon. Technol. Lett., vol. 8, no. 5, pp. 712-714, May 1996.

[29] T. Durhuus, C. Joergensen, B. Mikkelsen, R. J. S. Pedersen, and K. E. Stubkjaer, "All-optical data wavelength conversion by TWSOA's in a Mach-Zehnder configuration," IEEE Photon. Technol. Lett., vol. 6, pp. 53-55, 1994.

[30] P. S. Cho, D. Mahgerefteh, and J. Goldhar, " $10 \mathrm{~Gb} / \mathrm{s}$ RZ to NRZ format conversion using a semiconductor-optical-amplifier/fiber-Bragg-grating wavelength converter," in Proc. Eur. Conf. Opt, Commun, (ECOC 1998), Madrid, Spain, pp. 353-354.

[31] G.-R. Lin, P.-S. Hsueh, H.-H. Wu, and Y.-S. Liao, "The detuning characteristics of rational harmonic mode-locked semiconductor optical amplifier fiber-ring laser using backward optical sinusoidal-wave injection modulation," J. Lightw. Technol., vol. 23, pp. 1325-1333, 2005.

[32] D. Norte and A. E. Willner, "Demonstration of an all-optical data forma transparent WDM-to-TDM network node with extinction ratio enhancement for reconfigurable WDM networks," IEEE Photon. Technol. Lett., vol. 8, no. 5, pp. 715-717, May 1996.

[33] A. Reale, P. Lugli, and S. Betti, "Format conversion of optical data using four-wave mixing in semiconductor optical amplifiers," IEEE J. Sel.Top. Quantum Electron., vol. 7, pp. 703-709, Jul./Aug. 2001.

[34] L. X. Wang, B. C. Baby, V. Glesk, and I. Prucnal, "All-optical data format conversion between RZ and NRZ based on a Mach-Zehnder interferometric wavelength converter," IEEE Photon. Technol. Lett., vol. 15, no. 2, pp. 308-310, Feb. 2003.

[35] C. G. Lee, Y. J. Kim, C. S. Park, H. J. Lee, and C.-S. Park, "Experimental demonstration of $10-\mathrm{Gb} / \mathrm{s}$ data format conversions between $\mathrm{NRZ}$ and RZ 
using TWSOA-loop-mirror,” J. Lightw. Technol., vol. 23, pp. 834-841, 2005.

[36] G.-R. Lin, K.-C. Yu, and Y.-C. Chang, "10 Gbit/s all-optical non-returnto-zero to return-to-zero data format conversion based on a backward inverse-optical-comb injected semiconductor optical amplifier," Opt. Lett., vol. 31, pp. 1376-1378, 2006.

[37] I. D. Henning, M. J. Adams, and J. V. Collins, "Performance predictions from a new optical amplifier model," IEEE J. Quantum Electron., vol. 21, pp. 609-613, 1985.

[38] T. Durhuus, B. Mikkelsen, C. Joergensen, S. L. Danielsen, and K. E. Stubkjaer, "All-optical wavelength conversion by semiconductor optical amplifiers," J. Lightw. Technol., vol. 6, pp. 942-954, 1996.

[39] G.-R. Lin, K.-C. Yu, and Y.-C. Chang, "All-optical pulse data generation in a semiconductor optical amplifier gain-controlled by a reshaped optical clock injection," Appl. Phys. Lett., vol. 88, pp. 1114-1116, 2006.

[40] G. P. Agrawal and N. A. Olsson, "Self-phase modulation and spectral broadening of optical pulses in semiconductor laser amplifiers," IEEE J. Quantum Electron., vol. 25, no. 11, pp. 2297-2306, 1989.

[41] M. J. Connelly, Semiconductor Optical Amplifiers. Boston, MA: Kluwer, Ch. 5, 2000.

[42] G. P. Agrawal and N. A. Olsson, "Amplification and compression of weak picosecond optical pulses by using semiconductor laser amplifiers," Opt. Lett., vol. 14, pp. 500-502, 1989.

[43] A. E. Willner and W. Shieh, "Optimal spectral and power parameters for all-optical wavelength shifting: Single stage, fanout, and cascadability," J. Lightwave Technol., vol. 13, pp. 771-781, 1995.

[44] G. P. Agrawal, Fiber-Optic Communication Systems. New York: Wiley, Ch. 8, 1992.

[45] M. T. Hill, E. Tangdiongga, H. de Waardt, G. D. Khoe, and H. J. S. Dorren, "Carrier recovery time in semiconductor optical amplifiers that employ holding beams," Opt. Lett., vol. 27, pp. 1625-1627, 2002.

[46] F. Ginovart and J. C. Simon, "Wavelength dependence of gain recovery time in a semiconductor optical amplifier based wavelength shifter," in Proc. Conf. CLEO/Europe, Jun. 2003, p. 81.

[47] Y. Ben-Ezra, M. Haridim, and B. I. Lembrikov, "Theoretical analysis of gain-recovery time and chirp in QD-SOA," IEEE Photon. Technol. Lett., vol. 17, no. 9, pp. 1803-1805, Sep. 2005.

[48] J. L. Pleumeekers, M. Kauer, K. Dreyer, C. Burrus, A. G. Dentai, S. Shunk, J. Leuthold, and C. H. Joyner, "Acceleration of gain recovery in semiconductor optical amplifiers by optical injection near transparency wavelength," IEEE Photon. Technol. Lett., vol. 14, no. 1, pp. 12-14, Jan. 2002.

[49] F. Ginovart, M. Amaya, and A. Sharaiha, "Semiconductor optical amplifier studies under optical injection at the transparency wavelength in copropagative configuration," OSA/IEEE J. Lightw. Technol., vol. 25, no. 3, pp. 840-849, Mar. 2007.

[50] G. Walter, A. James, N. Holonyak, Jr., and M. Feng, "Chirp in a transistor laser: Franz-Keldysh reduction of the linewidth enhancement," Appl. Phys. Lett., vol. 90, pp. 1109-1111, 2007.
[51] T. Watanable, N. Sakaida, H. Yasaka, and M. Koga, "Chirp control of an optical signal using phase modulation in a semiconductor optical amplifier," IEEE Photon. Technol. Lett., vol. 10, no. 7, pp. 1027-1029, Jul. 1998.

[52] N. S. Bergano, F. W. Kerfoot, and C. R. Davidsion, "Margin measurements in optical amplifier system," IEEE Photon. Technol. Lett., vol. 5, no. 3, pp. 304-306, Mar. 1992.

[53] S. Sangbae, A. Byung-Gu, C. Mungweon, C. Seongdae, K. Daejeong, and P. Youngil, "Optics layer protection of Gigabit-Ethernet system by monitoring optical signal quality," Electron. Lett., vol. 38, pp. 1118$1119,2002$.

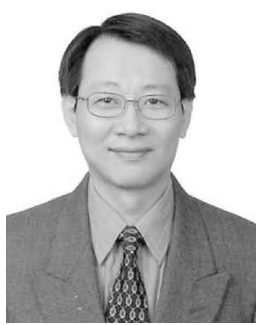

Gong-Ru Lin (S'93-M'96-SM'04) received the B.S. degree in physics from Soochow University, Taipei, Taiwan, in 1988, and the M.S. and Ph.D. degrees in electrooptical engineering from the $\mathrm{Na}$ tional Chiao Tung University (NCTU), Hsinchu City, Taiwan, in 1990 and 1996, respectively.

$\mathrm{He}$ is currently a Professor and the Vice Chairman at the Graduate Institute of Photonics and Optoelectronics, Department of Electrical Engineering, National Taiwan University, Taipei. His current research interests include nanocrystallite semiconductor physics and optoelectronics, high-speed fiber-optic communication systems, ultrafast lasers, and microwave/millimeter wave photonics. He is the author or coauthor of more than 100 papers published in international periodicals and more than 120 papers published in international conferences.

Prof. Lin has been the Vice Chair of ILLL Laser and Electro-Optics Society (IEEE/LEOS) Taipei Chapter since 2007 and the Fellow of the International Society for Optical Engineers (SPIE) since 2008.

Kun-Chieh Yu, photograph and biography not available at the time of publication.

Mu-Chieh Lo, photograph and biography not available at the time of publication. 